\title{
Low Mitotic Activity of Peripheral Lymphocytes During the First Two Years of Life
}

\author{
NICHOLAS MATSANIOTIS, CLEOPATRA ECONOMOU-MAVROU, \\ and CHRISTINE TSENGHI \\ From the Paediatric Clinic, Athens University, St. Sophie's Children's Hospital
}

It has long been a puzzle why there are so many lymphocytes normally circulating in the baby and the very young child. If the lymphocytes in infancy and early childhood are not fully developed in their immunological capacity, it is conceivable that more of these cells are produced in an attempt to compensate for their relative inadequacy.

In the present study an attempt was made to approach the problem of 'physiological' lymphocytosis in early life by investigating the lymphocyte response to phytohaemagglutinin (PHA) in children less than 2 years old.

\section{Patients and Method}

Mitotic counts were made in leucocyte cultures from 65 children less than 2 years of age, 21 children 8-13 years old, and 39 normal adults. The children studied were either normal or convalescing from mild upper respiratory infections.

Duplicate cultures were set up and examined for metaphase plates according to the method of Moorhead, Nowell, Mellman, Battips, and Hungerford (1960). Colchicine was added five hours before the end of a 72-hour incubation period. $0.2 \mathrm{ml}$. PHA (Wellcome) were used to induce mitosis. Mitotic counts were made in no less than 2000 cells.

In 5 of the younger and in 8 of the older children cultures were set without PHA. The proportion of cells undergoing mitosis never exceeded $0.1 \%$ in these cultures.

Received December 12, 1966.
In 6 children, mitotic counts were made twice over a period of a few days to a few weeks. The values obtained were constant.

\section{Results}

The results have been summarized in the Table. The mean mitotic index was lower in the children less than 2 years of age than in the older children or the adults. The difference in the mean mitotic index between children 8-13 years old and adults was statistically insignificant $(t=0.221<1.96$, $\mathrm{p}=0.05$ ); these older children and the adults were, therefore, grouped together as controls. The difference between the mean of children less than 2 years old and that of the controls was statistically highly significant $(t=7 \cdot 426>2 \cdot 626, \mathrm{p}<0.001)$.

There was considerable overlapping in the mitotic counts obtained from the two groups. However, in 33 out of 65 children less than 2 years old $(51 \%)$ mitotic counts were lower than the lowest count obtained from the controls. On the other hand, there were 21 children less than 2 years old (32\%) in whom mitotic counts lay within one standard deviation from the mean of the control group, in other words they were of the same order as those obtained from older people.

In the Figure, mitotic counts in the group of children less than 2 years old have been plotted against age. The coefficient of correlation was $0.425(\mathrm{p}<0.05)$ and the slope of the best line

TABLE

Mitotic Activity of Peripheral Lymphocytes

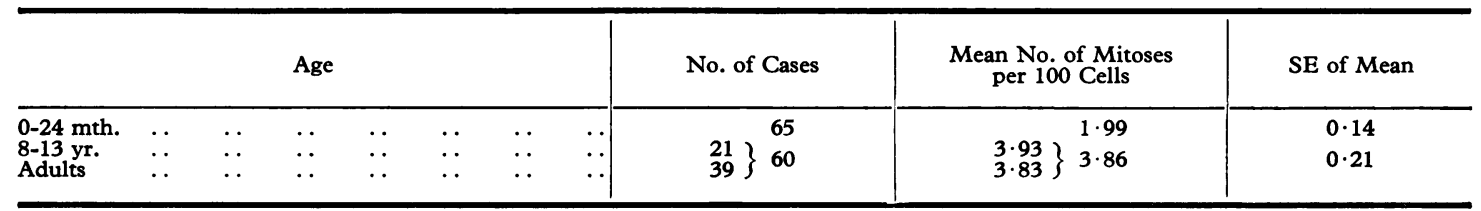




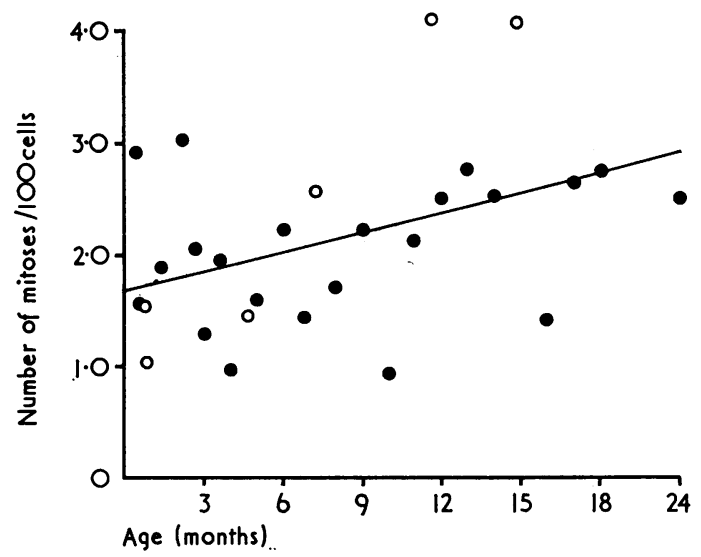

FIG.-Mitotic counts plotted against age in children less than 2 years old. $\bullet$, mean values from 2-5 subjects. $\bigcirc$, values from one subject.

drawn through the points calculated by the method of least squares suggests that the mitotic index increased with increasing age in these children.

\section{Discussion}

The mean mitotic index in children less than 2 years old was about one-half that of older children or adults. These results are not in agreement with those of Swedish workers who reported that in lymphocytes cultured from foetal, newborn, and adult blood the percentages of cells in mitosis were all in the same range (Lindahl-Kiessling and Böök, 1964). However, the experimental conditions of these workers were different from ours and the number of cases they studied in each group was very small.

The weaker lymphocytic response in the baby and the very young child cannot be thought to reflect its relative inexperience with antigen, since PHA is a ubiquitous mitogenic substance; it does not seem unreasonable to assume that the difference between the younger and the older subjects in their lymphocytic response to bacterial or viral antigen may be even greater than that observed with PHA.

The human baby is born with immunoglobulins which are almost entirely derived from his mother, but, if stimulated, he may form antibody before he is born (Lesser, 1927; Silverstein, 1962; Schaffer, 1965); he responds adequately to active immunization and appears to handle most viral and bacterial infections fairly well (Eichenwald, and Kotsevalov, 1960; Smith, Eitzman, Catlin, Wirtz, and Miller, 1964).
He can develop delayed hypersensitivity to tuberculin following BCG vaccination (Schaffer, 1965) and he can promptly reject homografts (Fowler, Schubert, and West, 1960).

On the other hand, the incidence and mortality of many infections, such as tuberculosis or whooping cough, are higher in infancy than in later life. If contracted in foetal life or at birth, tuberculosis runs a severe, overwhelming course; tuberculin hypersensitivity develops months after infection, if ever (Schaffer, 1965; Grady, and Zuelzer, 1955), and the tubercles formed in these babies are soft, with local necrosis and little cellular reaction (Schaffer, 1965). These observations, together with the weaker in vitro response of lymphocytes to PHA, are suggestive of relative incompetence of the cellular immune response of a considerable number of babies. This is corroborated by the recent observation that in mice two weeks after birth there is a tremendous increase of lymphocytes in the bone-marrow and that the circulating lymphocytes are mainly bonemarrow and lacking immunological qualities (J. M. Yoffey, 1966, personal communication). In fact, it is quite possible that bone-marrow lymphocytes are also predominant in the human baby and that they do not respond to PHA or antigen, at least in the same way as do cells constituting the lymphocytic population later in life.

The observation made in this study, that in some children less than 2 years old the lymphocytic response to PHA was of the same order as that of older people, suggests that individual variation in the capacity of the lymphocyte to proliferate under antigenic stimulation may reflect individual variation in natural resistance.

These preliminary results have certainly raised many questions rather than offered solutions to the puzzle of 'physiological' lymphocytosis in early life, which may prove not to be a purposeless extravagance of nature.

\section{Summary}

The in vitro response of peripheral lymphocytes to phytohaemagglutinin was investigated in children less than 2 years old, in children 8-13 years old, and in adults.

The mean mitotic index in the very young children was about one-half that of older children or adults. Mitotic counts tended to increase with increasing age during the first two years of life.

It is suggested that the weaker lymphocytic response to phytohaemagglutinin may be associated with relative incompetence of the cellular immune mechanism in early life. 
REFERENCES

Eichenwald, H. F., and Kotsevalov, O. (1960). Immunologic responses of premature and full-term infants to infection with certain viruses. Pediatrics, 25, 829.

Fowler, R., Jr., Schubert, W. K., and West, C. D. (1960). Acquired partial tolerance to homologous skin grafts in the human infant at birth. Ann. N.Y. Acad. Sci., 87, 403.

Grady, R. C., and Zuelzer, W. W. (1955). Neonatal tuberculosis. Amer. F. Dis. Child., 90, 381.

Lesser, F. (1927). Serologie der kongenitalen Syphilis. In Handbuch der Haut und Geschechtskrankheiten, ed. J. Jadassohn, vol. 19, p. 287. Springer, Berlin.

Lindahl-Kiessling, K., and Böök, J. A. (1964). Effects of phytohaemagglutinin on leucocytes. Lancet, 2, 591.
Moorhead, P. S., Nowell, P. C., Mellman, W. J., Battips, D. M., and Hungerford, D. A. (1960). Chromosome preparations of leukocytes cultured from human peripheral blood. Exp. Cell Res., 20, 613.

Schaffer, A. J. (1965). Disease of the Newborn. Saunders, Philadelphia and London.

Silverstein, A. M. (1962). Congenital syphilis and the timing of immunogenesis in the human foetus. Nature (Lond.), 194, 196.

Smith, R. T., Eitzman, D. V., Catlin, M. E., Wirtz, E. O., and Miller, B. E. (1964). The development of the immune response. Characterization of the response of the human infant and adult to immunization with Salmonella vaccines. Pediatrics, 33, 163. 